\title{
PLIN2 confers gefitinib resistance by inhibiting cell apoptosis via activation of EGFR/AKT/survivin in PC9R cells
}

\author{
Meijia Chang ${ }^{1,3, *}$, Jie Hu ${ }^{1,3, *}$, Tao Fang ${ }^{2, *}$, Jing $\mathrm{Li}^{1,3}$, Yuanlin Song ${ }^{1,3}$, Chunxue Bai ${ }^{1,3,4}$ \\ and Jian Zhou ${ }^{1,3}$ \\ ${ }^{1}$ Department of Pulmonary Medicine, Zhongshan Hospital, Fudan University, Shanghai, China \\ ${ }^{2}$ Department of Oncology, Shengli Oilfield Central Hospital, Dongying, China \\ ${ }^{3}$ Shanghai Respiratory Research Institute, Shanghai, China \\ ${ }^{4}$ State Key Laboratory of Respiratory Disease, Guangzhou Medical University, Guangzhou, China \\ *These authors contributed equally to this work \\ Correspondence to: Jian Zhou, email: zhou.jian@fudan.edu.cn \\ Chunxue Bai, email: bai.chunxue@zs-hospital.sh.cn
}

Keywords: non-small cell lung cancer; PLIN2; gefitinib; apoptosis; TKI resistance

Received: November 01, $2017 \quad$ Accepted: January 04, $2018 \quad$ Published: January 10, 2018

Copyright: Chang et al. This is an open-access article distributed under the terms of the Creative Commons Attribution License 3.0 (CC BY 3.0), which permits unrestricted use, distribution, and reproduction in any medium, provided the original author and source are credited.

\section{ABSTRACT}

Epidermal growth factor receptor (EGFR) tyrosine kinase inhibitors (TKIs) are the first-line drugs for advanced non-small cell lung cancer (NSCLC) patients with activating EGFR mutations. However, the development of TKI resistance is a major clinical challenge. Here we show that the adipophilin/adipose differentiation-related protein perilipin (PLIN)2 was upregulated in gefitinib-resistant PC9R cells; PLIN2 knockdown in PC9R cells conferred gefitinib resistance by inhibiting cancer cell apoptosis in vitro and in vivo and by inducing mitochondrial dysfunction and caspase activation. PLIN2 was also shown to activate EGFR/AKT/survivin signalling and was overexpressed in gefitinib-resistant cells in clinical samples. These results indicate that PLIN2 confers gefitinib resistance in lung cancer by inhibiting apoptosis and activating the EGFR/AKT/survivin pathway, and is thus a potential therapeutic target in EGFR TKI-resistant NSCLC patients.

\section{INTRODUCTION}

Lung cancer is a highly aggressive malignancy and the leading cause of cancer-related death worldwide, with non-small cell lung cancer (NSCLC) being one of the most common subtypes. First-line chemotherapy for NSCLC patients with advanced disease is typically platinum-based, and yields a median overall survival of 8-11 months [1]. About $10 \%$ of NSCLC patients have a mutation in the tyrosine kinase (TK) domain of the epidermal growth factor receptor (EGFR) gene, and the success of EGFR TK inhibitors (TKIs) has shifted the focus of cancer treatment from cytotoxic chemotherapy to one that targets specific molecules. In fact, EGFR TKIs are now the standard therapy for advanced NSCLC patients with activating EGFR mutations [2]. However, approximately 20\% to
$30 \%$ of NSCLC patients are unresponsive to these drugs and even those who initially benefit from therapy develop progressive disease after about 1 year [3-4]. Thus, many efforts have been made to clarify EGFR TKI resistance mechanisms, which include a secondary mutation in T790M in exon 20 of the EGFR gene [5], amplification of the MET proto-oncogene [6], and hepatocyte growth factor (HGF) overexpression [7]. However, the mechanistic basis for resistance is unknown in $20 \%-30 \%$ of cases.

Lipid metabolism plays an important role in lung cancer. The adipophilin/adipose differentiation-related protein perilipin (PLIN)2 is a typical member of the adipophilin family of lipid droplet (LD) coat proteins that regulate lipid storage and lipolysis. PLIN1 is primarily expressed in adipose and steroidogenic cells, while PLIN2 is ubiquitously expressed and functions as the predominant 
LD coat protein in non-adipose tissues [8], playing a key role in fatty acid uptake, LD formation, and lipid storage. Previous studies have shown that PLIN2 is upregulated in many cancers, including clear cell renal cell carcinoma (ccRCC), malignant melanoma, and colorectal cancer [9-10]. Hypoxia-inducible factor (HIF)-2 $\alpha$ was found to promote PLIN2 expression and lipid storage in ccRCC cell lines, which was required for endoplasmic reticulum (ER) homeostasis and resistance to cytotoxic ER stress [11].

PLIN2 was found to be expressed in a subset $(15.5 \%)$ of lung adenocarcinoma cases. The expression level was significantly correlated with vascular invasion, wild-type EGFR expression, anaplastic lymphoma kinase fusion, and surfactant protein B deficiency. Furthermore, patients with PLIN2-positive adenocarcinoma had worse disease-free and overall survival than those who were negative for PLIN2 expression [12]. However, the precise function of PLIN2 in lung adenocarcinoma has not yet been reported.

We addressed this in the present study using gefitinib-resistant PC9R cells and clinical samples. We found that PLIN2 was upregulated in PC9R cells and conferred gefitinib resistance by inducing cell cycle arrest and apoptosis. PLIN2 knockdown in PC9R cells impaired the activation of AKT signalling. Additionally, PLIN2 was overexpressed in gefitinib-resistant cells of human adenocarcinoma specimens.

\section{RESULTS}

\section{PLIN2 is upregulated in gefitinib-resistant PC9R cells and mediates gefitinib resistance}

Based on previous studies, we speculated that PLIN2 plays an important role in gefitinib resistance. We generated gefitinib-resistant PC9R cells by exposing gefitinib-sensitive PC9 cells to increasing concentrations of gefitinib [13]. To investigate a role for PLIN2 in the development of gefitinib resistance, we compared PLIN2 expression levels between gefitinib-sensitive PC9 and -resistant PC9R cells by quantitative reverse transcription (qRT)-PCR and western blotting. We found that PLIN2 was upregulated in PC9R as compared to PC9 cells (Figure 1A-1C), suggesting an important role for PLIN2 in gefitinib resistance.

To evaluate this possibility, PC9R cells were transfected with either PLIN2 short hairpin (sh) RNA or negative control shRNA to silence PLIN2 expression. The qRT-PCR and western blot analyses showed that shPLIN2-1 and -2, two shRNA sequences targeting the PLIN2 transcript, inhibited PLIN2 expression (Figure 1D1F). PC9R cells were transfected with PLIN2 or negative control shRNA for $24 \mathrm{~h}$ and then treated with $1 \mu \mathrm{M}$ gefitinib for $96 \mathrm{~h}$. PLIN2 knockdown with shPLIN2-1 and -2 reduced cell proliferation (Figure $1 \mathrm{G}$ ), as confirmed with the Cell Counting Kit (CCK) 8 cell viability assay for gefitinib concentrations ranging from 0.1 to $20.0 \mu \mathrm{M}$ (Figure 1H).

\section{PLIN2 confers gefitinib resistance by inhibiting PC9R cell apoptosis through caspase activation and mitochondrial dysfunction}

We investigated the effects of PLIN2 silencing on PC9R cell proliferation following gefitinib treatment by examining 5-ethynyl-2'-deoxyuridine (EdU) incorporation. The fraction of EdU-positive cells was approximately $65 \%$ in the negative control group and 35\% in cells transfected with shPLIN2-1 and -2 following incubation with $1 \mu \mathrm{M}$ gefitinib (Figure 2A, 2B), confirming that PLIN2 depletion suppresses proliferation in PC9R cells.

To further verify the effects of PLIN2 silencing on gefitinib resistance in PC9R cells, we analysed apoptosis by annexin V-fluorescein isothiocyanate (FITC)/propidium iodide (PI) staining and flow cytometry. The apoptosis rate was increased to $\sim 40 \%$ in PC9R cells transfected with shPLIN2-1 and -2 as compared to negative control shRNA in the presence of $1 \mu \mathrm{M}$ gefitinib (Figure 2C, 2D). Moreover, the number of cells in G2/M phase was increased in PLIN2-silenced PC9R cells relative to the control group, indicating that cell cycle arrest was induced (Figure 2E, 2F). To confirm the effects of PLIN2 silencing in both PC9 and PC9R cells in the absence of gefitinib, we analysed apoptosis of PLIN2-silenced PC9 and PC9R cells by FITC/PI staining and flow cytometry. The qRT-PCR analyses confirmed that shPLIN2-1 and -2 significantly inhibited PLIN2 expression in the PC9 cells (Supplementary Figure 1A). The apoptosis rate was increased to $\sim 20 \%$ in both PC9 and PC9R cells transfected with shPLIN2-1 and -2 as compared to negative control shRNA in the absence of gefitinib (Supplementary Figure $1 \mathrm{~B}, 1 \mathrm{C})$; however, the increase in the apoptosis rate of PLIN2-silenced PC9R cells in the absence of gefitinib was small compared to that in the presence of $1 \mu \mathrm{M}$ gefitinib.

We next measured mitochondrial membrane potential by staining cells that were stably transfected with PLIN2 or negative control shRNA with JC-1, which emits red fluorescence as J-aggregates in intact mitochondria and green fluorescence in the monomeric form in damaged mitochondria. The JC-1 monomer ratio of PC9R cells transfected with shPLIN2-1 and -2 was higher than that of cells transfected with the negative control shRNA in the presence of $1 \mu \mathrm{M}$ gefitinib (Figure 3A, 3B). The generation of reactive oxygen species (ROS) was increased by nearly 6 fold in PC9R cells transfected with shPLIN2-1 and -2 as compared to the negative control group upon treatment with $1 \mu \mathrm{M}$ gefitinib, as determined by CellROX Orange staining (Figure 3C, 3D). Moreover, the apoptotic proteins cleaved Caspase-3 and cleaved poly-ADP-ribose polymerase (PARP) were upregulated in PLIN2-depleted as compared to control cells (Figure 3E-3G). 


\section{PLIN2 knockdown suppresses tumour proliferation in vivo}

To determine whether PLIN2 knockdown suppresses lung tumour growth in vivo, PC9R cells transfected with shPLIN2-1 were injected into nude mice. After 1 week, PC9R cells transfected with negative control and shPLIN2-1 grew into visible tumours. However, the volume of tumours derived from cells transfected with shPLIN2-1 was significantly smaller than that of tumours derived from negative control shRNA-transfected cells (Figure 4A). A similar trend was observed for tumours mass after 1 month (Figure 4B, 4C). To assess the effects of PLIN2 knockdown on cell proliferation, Ki67 expression in tumour sections was detected by immunohistochemistry. The percentage of Ki67-positive cells was decreased in PLIN2-deficient PC9R cell tumours relative to the negative control (Figure 4D, 4E).
A

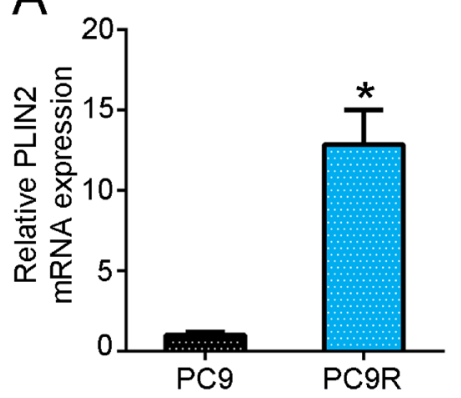

D

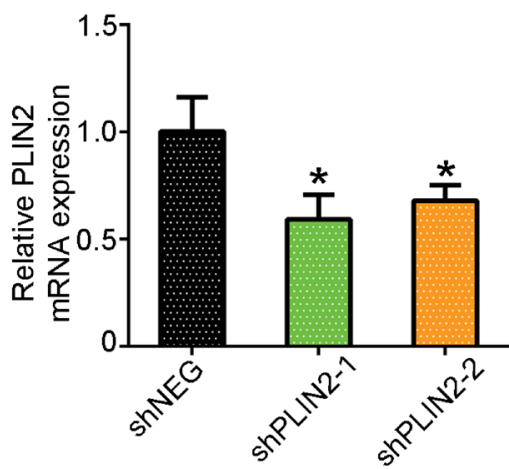

E

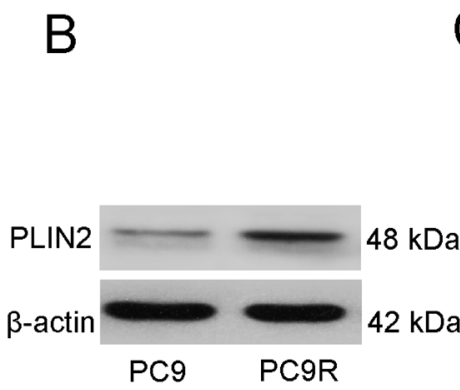

PLIN2

$\beta$-actin

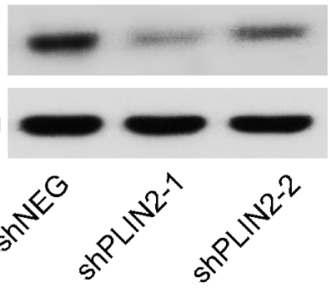

C

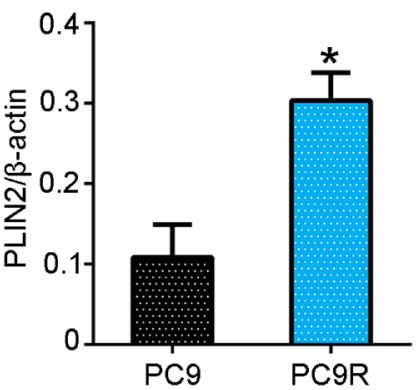

$\mathrm{F}$

G

PC9R(1 $1 \mu M$ Gefitinib)
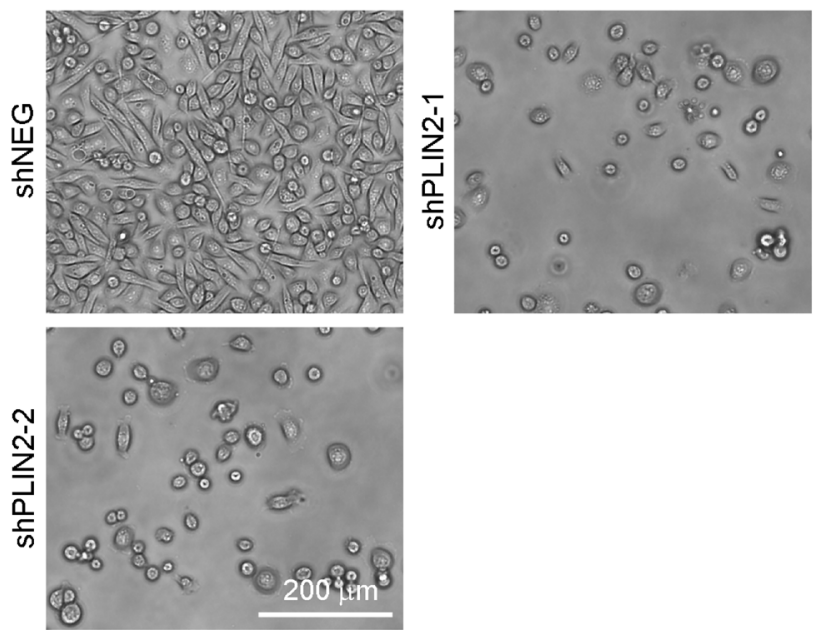

$\mathrm{H}$
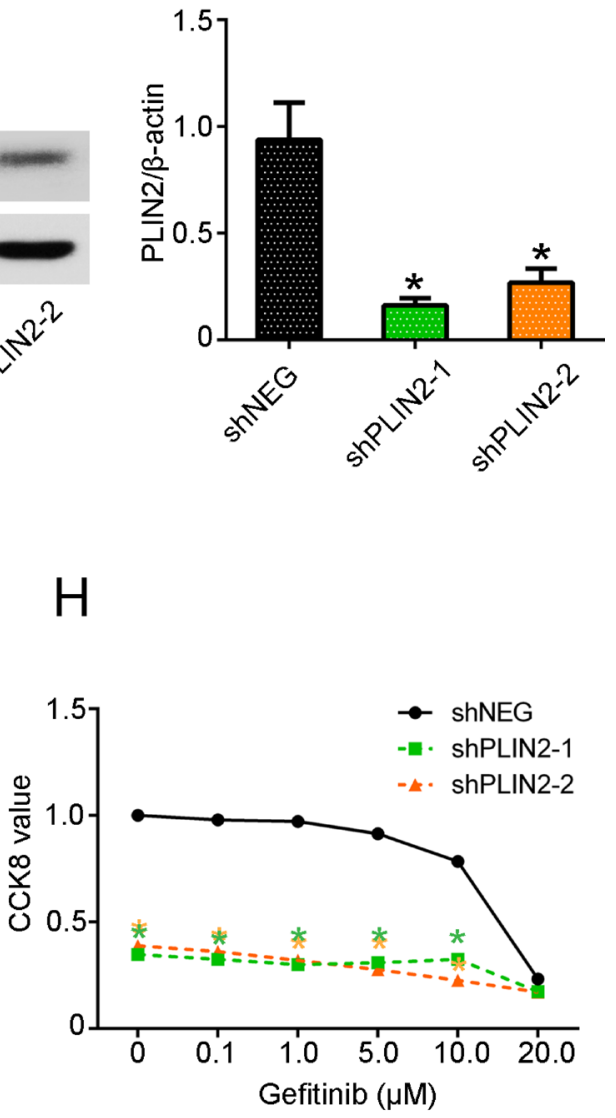

Figure 1: PLIN2 is upregulated in gefitinib-resistant PC9R cells and confers gefitinib resistance. (A) PLIN2 expression in PC9 and PC9R cells was detected by qRT-PCR. (B, C) Western blot analysis of PLIN2 protein levels in PC9 and PC9R cells. (D) qRTPCR detection of PLIN2 mRNA levels in PC9R cells transfected with shPLIN2 or negative control shRNA (shNEG). (E, F) Western blot analysis of PLIN2 protein levels in PC9R cells transfected with shPLIN2 or shNEG. (G) Morphological analysis of PC9R cells transfected with shPLIN2 or shNEG and cultured in the presence of $1 \mu \mathrm{M}$ gefitinib. (H) Viability of PC9R cells transfected with shPLIN2 or shNEG and treated with different concentrations of gefitinib, as determined with CCK8. ${ }^{*} P<0.05$ vs. negative control. 


\section{PLIN2 knockdown inhibits PC9R cell proliferation by suppressing EGFR/AKT/survivin signalling}

To evaluate the molecular mechanism underlying PLIN2-mediated gefitinib resistance in PC9R cells, we used a whole human cDNA array to identify altered downstream signalling pathways. PLIN2 depletion altered the expression of apoptosis and cell cycleassociated molecules. A qRT-PCR analysis revealed that Survivin (also called baculoviral inhibitor of apoptosis repeat-containing 5 or BIRC5), DNA topoisomerase 2-alpha (TOP2A), cyclin-dependent kinase (CDK)1, and B cell lymphoma 2 ligand (BCL2L)1 were significantly downregulated in cells transfected with shPLIN2-1 as compared to those transfected with the negative
A

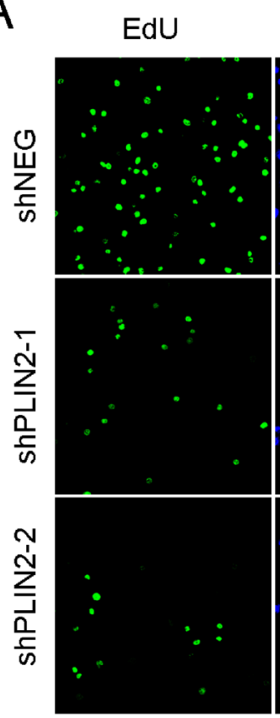

C

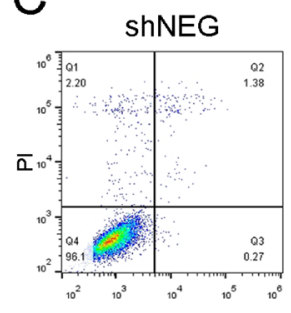

Annexin V - FITC

E

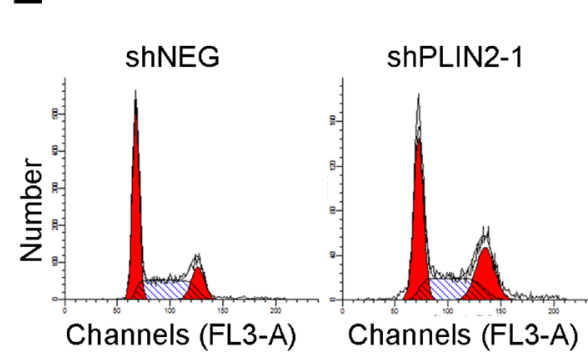

Hoechst

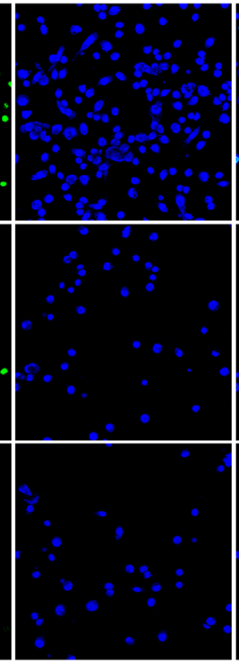

ShPLIN2-1

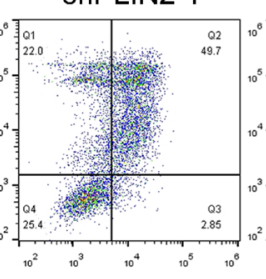

Annexin V - FITC

Annexin V - FITC
B
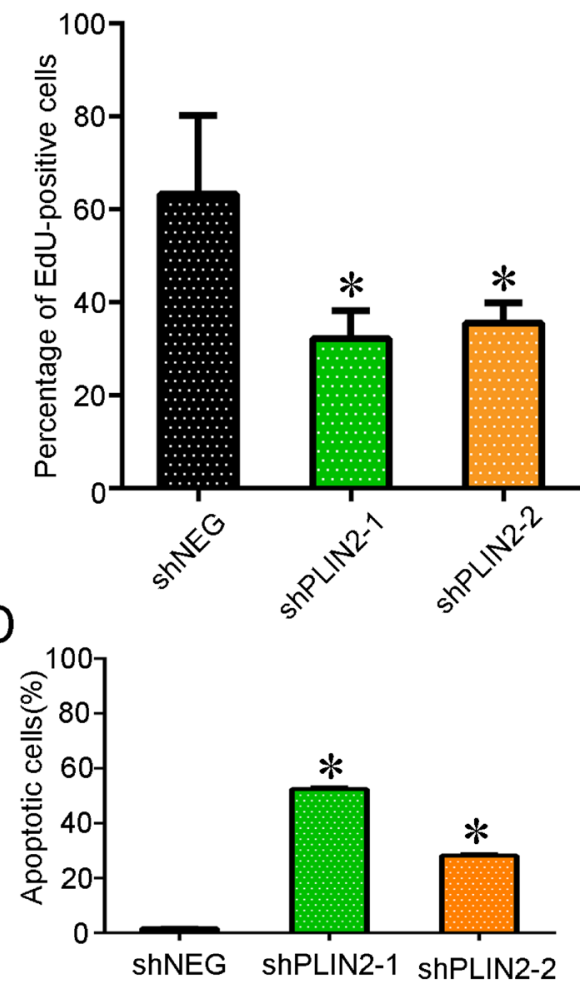

F

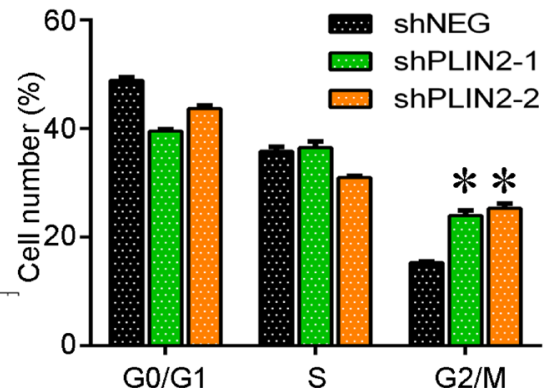

Figure 2: PLIN2 confers gefitinib resistance by inhibiting PC9R cell apoptosis and cell cycle arrest. (A, B) PC9R cells transfected with PLIN2 shRNA or negative control shRNA (shNEG) were treated with $1 \mu \mathrm{M}$ gefitinib and then stained with EdU and Hoechst 33342. The percentage of EdU-positive cells was higher in the negative control group than in the shPLIN2-1 and -2 groups. Data are representative of at least three similar experiments. (C, D) PC9R cells transfected with PLIN2 shRNA or shNEG were treated with $1 \mu \mathrm{M}$ gefitinib, stained with FITC-annexin V/PI, and then analysed by flow cytometry. Transfection of shPLIN2-1 or shPLIN2-2 increased apoptosis in PC9R cells relative to the negative control. (E, F) Detection of cells in G1, S, and G2 phases of the cell cycle by flow cytometry. PC9R cells transfected with PLIN2 shRNA or shNEG were treated with $1 \mu \mathrm{M}$ gefitinib, and the number of cells in the different stages of the cell cycle was detected by flow cytometry. Data are representative of three similar experiments. ${ }^{*} P<0.05$ vs. negative control. 
control shRNA (Figure 5A, 5B). Given that AKT plays an important role in tumour cell proliferation, we investigated the relationship between the expression of PLIN2 and AKT signalling pathway components by western blotting. EGFR, AKT, phosphorylated (p-) $\mathrm{AKT}$, and survivin levels were markedly decreased in cells transfected with shPLIN2-1 or -2 (Figure 5C). Overexpression of EGFR partly reversed the apoptosis induced by PLIN2 depletion (Figure 5D). YM155 is a survivin inhibitor that has been investigated as an anticancer agent in clinical trials; Treatment with YM155 decreased the proliferation of PC9R cells relative to the negative control, especially in cells transfected with shPLIN2-1 or -2 (Figure 5E).

\section{PLIN2 overexpression increases EGFR TKI resistance in patients with lung cancer}

To confirm the role of PLIN2 in EGFR TKI resistance, we collected cancer cells from 26 malignant pleural effusions of lung adenocarcinoma (including 17 that were sensitive and nine that were resistant to EGFR TKI) for analysis of PLIN2 expression by qRT-PCR. The clinical characteristics of the 26 patients are listed in Table 1. There were no differences in the clinical characteristics between the sensitive group and resistant group. PLIN2 was downregulated in EGFR TKI-sensitive as compared to -resistant samples in EGFR-mutant lung adenocarcinomas (Figure 6A).
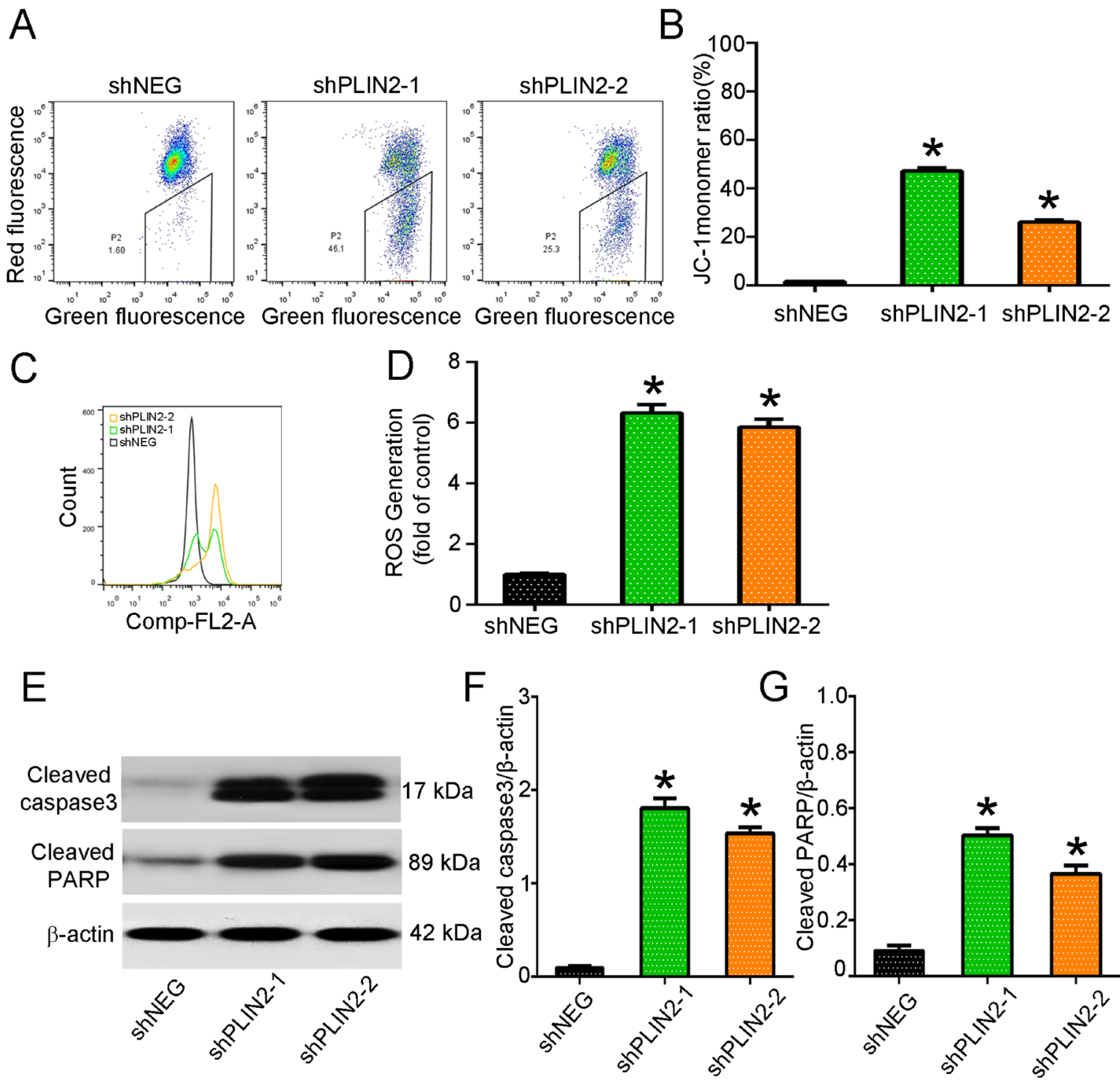

Figure 3: PLIN2 inhibits PC9R cell apoptosis by inducing mitochondrial dysfunction and caspase activation in the presence of gefitinib. (A, B) Representative histograms of JC-1 staining detected by flow cytometry. ShPLIN2-1 or -2 transfection increased the JC-1 monomer ratio in PC9R cells as compared to the negative control (shNEG) following incubation with $1 \mu \mathrm{M}$ gefitinib. (C, D) Detection of ROS by flow cytometry. ShPLIN2-1 or -2 increased ROS generation in PC9R cells relative to shNEG following incubation with $1 \mu \mathrm{M}$ gefitinib. (E-G) Western blot analysis of cleaved caspase-3 and cleaved PARP protein levels in PC9R cells transfected with shPLIN2 or shNEG. ${ }^{*} P<0.05$ vs. negative control. 


\section{DISCUSSION}

First-generation EGFR TKIs including gefitinib and erlotinib inhibit EGFR signalling by blocking the intracellular TK domain and are the first-line treatment for advanced NSCLC with EGFR mutation, especially in Asians, females, never smokers, and/or patients with adenocarcinoma [14]. However, approximately $20 \%-30 \%$ of NSCLC patients with EGFR mutation do not respond to treatment, and even those who show initial improvement eventually develop progressive disease about 1 year later [15]. Therefore, it is important to clarify the mechanisms of TKI resistance for more effective treatment of lung cancer.

In this study we found that PLIN2 is upregulated in gefitinib-resistant PC9R cells and confers gefitinib resistance. PLIN2 is a member of the PLIN family that

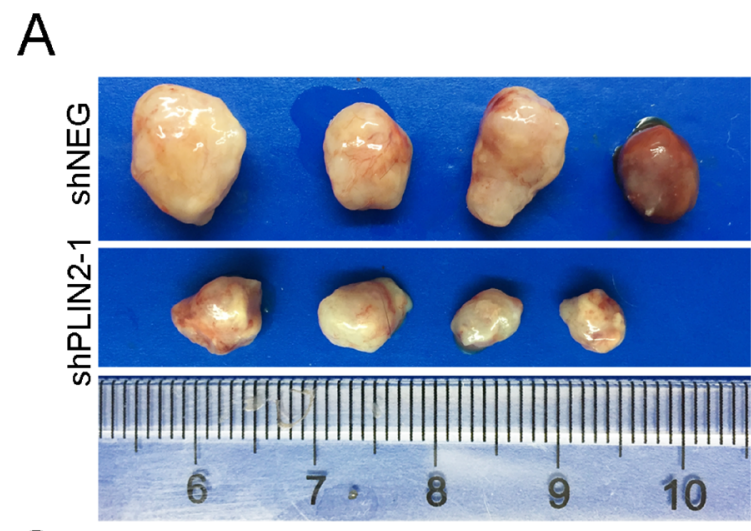

C
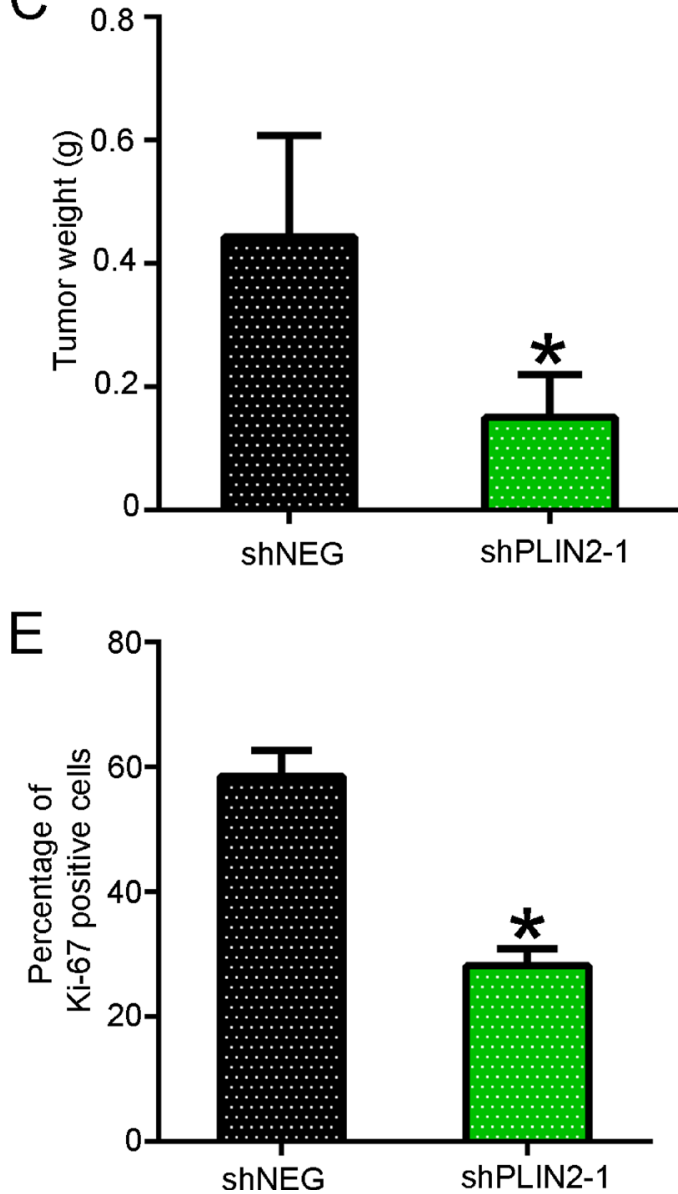

B

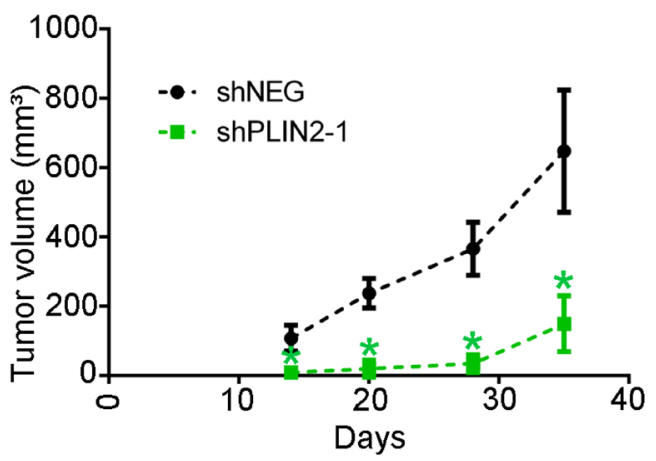

D

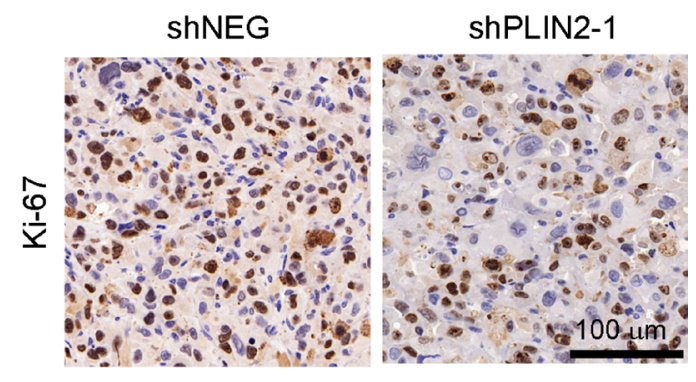

Figure 4: PLIN2 knockdown suppresses tumour cell proliferation in vivo. (A) Representative images of tumour tissues dissected from animals 1 month after injection of PC9R cells transfected with shPLIN2-1 or negative control (shNEG). (B) Tumour volume was measured twice a week. (C) Quantitative analysis of the weight of tumours derived from shPLIN2-1 or shNEG. (D) Representative images of Ki67 expression in xenograft tumours. (E) The Ki67-positive cells were quantified in randomly selected fields of each tissue section. There were five nude ( $n=5$ mice per group). ${ }^{*} P<0.05$ vs. negative control. 
plays an important role in fatty acid uptake, LD formation, and lipid storage [16]. Lung tumours exhibit abnormalities in vessels structure that limit the supply of nutrients and leads to hypoxia; therefore, metabolic processes such as carbohydrate and lipid metabolism must be altered in order to support cell growth [17]. Indeed, lipid metabolism has been implicated in lung cancer progression [18], while other studies have reported that PLIN2 is overexpressed in breast and colorectal cancers and malignant melanoma
[9-10] and promoted ccRCC proliferation by inhibiting ER stress through increased lipid storage [11]. PLIN2 expression in a subset of lung adenocarcinoma cases is associated with the presence of wild-type EGFR and reduced overall survival [12]. Thus, PLIN2 plays an important role in lung cancer progression, especially in TKI-resistant cases.

Cancer cell proliferation and survival depend on EGFR activation [19]. EGFR-TKIs induce cancer cells

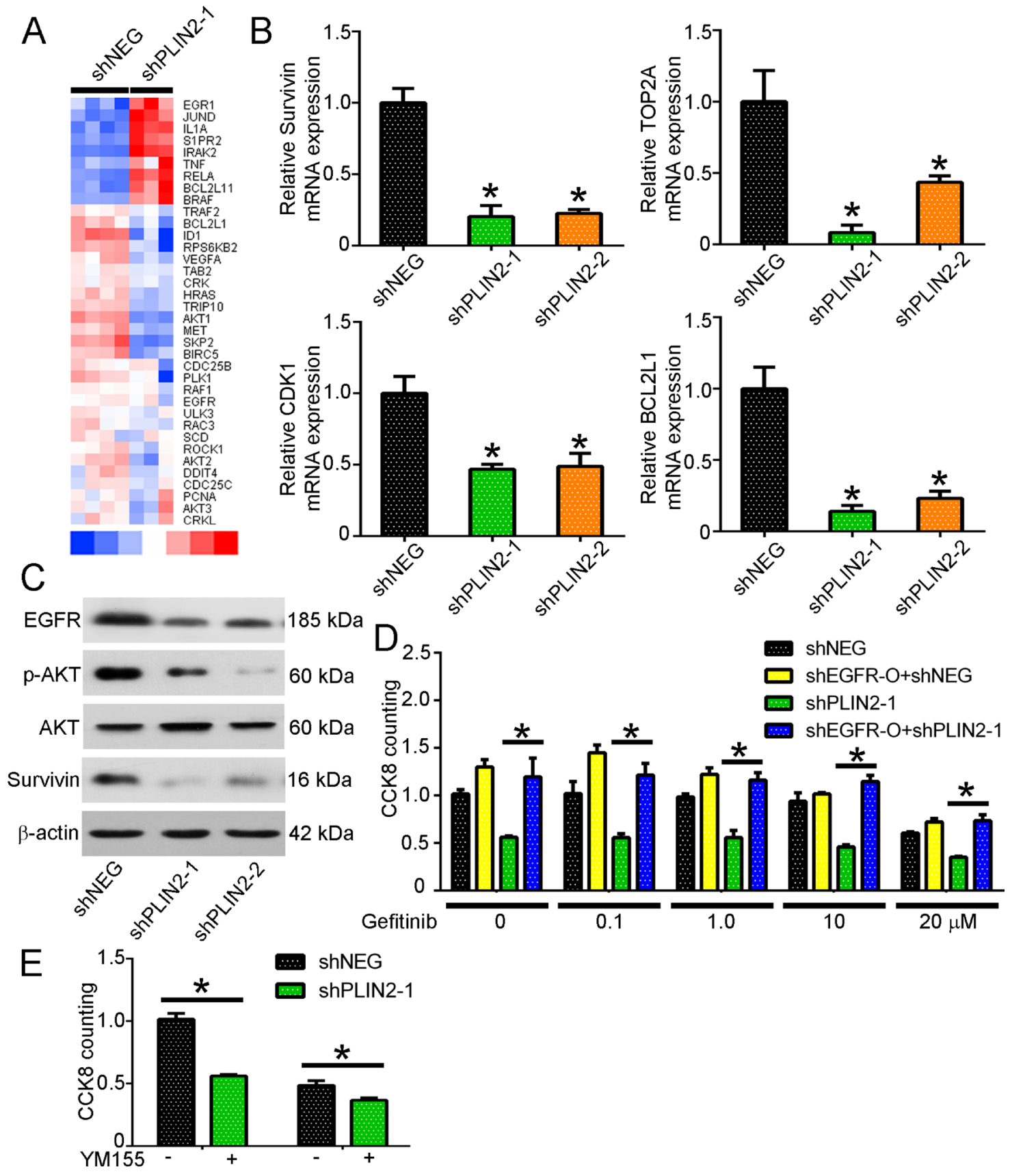

Figure 5: PLIN2 knockdown suppresses PC9R cell proliferation by inhibiting EGFR/AKT/survivin signalling. (A) Whole human cDNA array analysis. (B) Survivin, TOP2A, CDK1, and BCL2L1 levels in PC9R cells, as determined by qRT-PCR. (C) Western blot analysis of EGFR, p-AKT, AKT, and survivin protein levels in PC9R cells transfected with shPLIN2 or negative control shRNA (shNEG). (D) Proliferation of PC9R cells co-transfected with EGFR, shPLIN2, and shNEG, as determined by CCK8. (E) Proliferation of PC9R cells transfected with shPLIN2 and shNEG and treated with YM155, as determined with CCK8. ${ }^{*} P<0.05$ vs. negative control. 
Table 1: The clinical characteristics of EGFR TKI-sensitive and -resistance lung adenocarcinoma patients

\begin{tabular}{|c|c|c|c|c|c|}
\hline $\begin{array}{l}\text { Clinicopathological } \\
\text { Features }\end{array}$ & & $\begin{array}{c}\text { Number of } \\
\text { cases }\end{array}$ & $\begin{array}{c}\text { EGFR TKI- } \\
\text { sensitive }\end{array}$ & $\begin{array}{l}\text { EGFR TKI- } \\
\text { resistance }\end{array}$ & $p$ Value \\
\hline \multicolumn{6}{|l|}{ Age (years) } \\
\hline & $<65$ & 14 & 10 & 4 & 0.487 \\
\hline & $\geq 65$ & 12 & 7 & 5 & \\
\hline \multicolumn{6}{|l|}{ Gender } \\
\hline & Male & 9 & 4 & 5 & 0.102 \\
\hline & Female & 17 & 13 & 4 & \\
\hline \multicolumn{6}{|l|}{ Smoking } \\
\hline & No-smokers & 20 & 14 & 6 & 0.668 \\
\hline & Smokers & 5 & 3 & 2 & \\
\hline \multicolumn{6}{|l|}{ Tumor size } \\
\hline & $\mathrm{T} 1 / \mathrm{T} 2$ & 15 & 10 & 5 & 0.842 \\
\hline & $\mathrm{T} 3 / \mathrm{T} 4$ & 8 & 5 & 3 & \\
\hline \multicolumn{6}{|l|}{ lymphatic metastasis } \\
\hline & Absent & 15 & 9 & 6 & 0.611 \\
\hline & Present & 11 & 8 & 3 & \\
\hline \multicolumn{6}{|l|}{ Clinical stage } \\
\hline & IV 期 & 26 & 17 & 9 & \\
\hline
\end{tabular}

$P$ value represents the probability from a Chi-square test for different number of EGFR TKI-sensitive and -resistance cases.

apoptosis by blocking EGFR signalling. The T790M EGFR mutation is a major factor in the mechanism of EGFR TKI resistance. Furthermore, gene amplifications or mutations that cause growth factor receptor signalling downstream of EGFR, including $\mathrm{HGF} / \mathrm{c}-\mathrm{Met}$ or insulin-like growth factor 1 receptor, protect cancer cells against EGFR TKIinduced apoptosis $[2,20]$. EGFR-TKIs decreased cancer cell survival by blocking downstream signalling pathways such as mitogen-activated protein kinase/extracellular signal-regulated kinase, Janus kinase/signal transducer and activator of transcription, and phosphoinositide 3-kinase/AKT/mammalian target of rapamycin [21-24]. The EGFR/AKT/survivin pathway plays an important role in the induction of apoptosis by EGFR-TKIs in

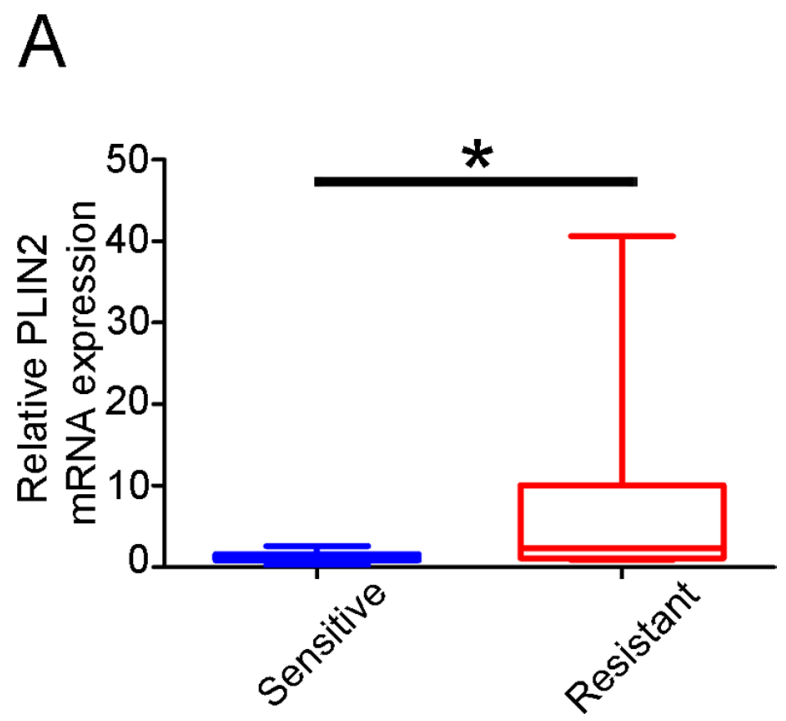

Figure 6: PLIN2 overexpression increases EGFR TKI resistance in lung cancer patients. (A) Scatterplot of $P L I N 2$ mRNA expression in lung adenocarcinomas sensitive or resistant to EGFR TKI. ${ }^{*} P<0.05$ vs. negative control. 
EGFR-mutant NSCLC cells [25]; we found that PLIN2 knockdown repressed the EGFR/AKT/survivin signalling and promoted apoptosis in gefitinb-resistant cancer cells. PLIN2 overexpression protected cancer cells against apoptosis induced by gefitinb, whereas PLIN2 knockdown activated apoptosis-associated proteins such as cleaved caspase- 3 and cleaved PARP.

In conclusion, we report here for the first time that PLIN2 is upregulated in gefitinb-resistant NSCLC and conferred gefitinb resistance by blocking gefitinb-induced apoptosis and activating EGFR/AKT/survivin signalling. Thus, PLIN2 plays an important role in the mechanism of resistance against EGFR-TKIs and is a potential target for the treatment of EGFR-TKI-resistant lung cancer.

\section{MATERIALS AND METHODS}

\section{Cell culture}

PC9 human lung adenocarcinoma cells were purchased from the American Type Culture Collection (Manassas, VA, USA). To induce gefitinib resistance, the cells were continuously exposed to increasing concentrations of gefitinib [13]. Gefitinib-sensitive and -resistant cells were cultured in Roswell Park Memorial Institute 1640 medium (Thermo Fisher Scientific, Waltham, MA, USA) supplemented with 10\% heatinactivated fetal bovine serum and $100 \mathrm{U} / \mathrm{ml}$ penicillin/ streptomycin. Gefitinib resistance was maintained by adding $1 \mu \mathrm{M}$ gefitinib (Selleckchem, Houston, TX, USA). The cells were grown as a monolayers in a humidified atmosphere of $5 \% \mathrm{CO}_{2}$ at $37^{\circ} \mathrm{C}$.

\section{Lentivirus construction and infection}

ShRNAs against PLIN2 (shPLIN2-1 and shPLIN2-2) were obtained from The RNAi Consortium (Broad Institute, Cambridge, MA, USA). Lentiviral plasmids containing GV112-shPLIN2-1 or -shPLIN2-2 of negative control shRNA were obtained from GeneChem (Shanghai, China). Lentiviral vectors overexpressing human EGFR (GV358) (GenBank accession number NM_005228) were from GeneChem. Lentiviral particles were produced by transfecting HEK 293T cells with the vectors. For viral infection, PC9 or PC9R cells were seeded in 6-well plates and grown to $50 \%-70 \%$ confluence, then incubated in medium containing the virus and $4 \mu \mathrm{g} / \mathrm{ml}$ polybrene at a multiplicity of infection of 10 , followed by incubation with various concentrations of gefitinib $24 \mathrm{~h}$ later.

\section{Cell proliferation assay}

CCK8 (Dojindo Laboratories, Kumamoto, Japan) was used to assess the rate of cell proliferation. Briefly, cells were seeded in 96-well plates at approximately 1000 cells/well in $200 \mu \mathrm{l}$ of culture medium. After $24 \mathrm{~h}$, $10 \mu \mathrm{l}$ CCK8 solution was added to each well, and the plates were incubated for $1 \mathrm{~h}$ at $37^{\circ} \mathrm{C}$. The absorbance at $450 \mathrm{~nm}$ was determined on a Multiskan microplate reader (Thermo Fisher Scientific) at a wavelength of $650 \mathrm{~nm}$.

\section{EdU incorporation assay}

Cells were incubated with $10 \mu \mathrm{M}$ EdU (Thermo Fisher Scientific) for $4 \mathrm{~h}$ and then fixed with $3.7 \%$ formaldehyde in phosphate-buffered saline (PBS) for $15 \mathrm{~min}$ at room temperature. EdU detection was performed according to manufacturer's instructions and samples were imaged on an A1R confocal laser scanning microscope system (Nikon, Tokyo, Japan). PC9R cells stained with Hoechst 33342 and positive for EdU were counted using ImageJ v. 1.42 software (National Institutes of Health, Bethesda, MD, USA).

\section{Detection of apoptotic cells by flow cytometry}

An annexin V-FITC/PI staining kit (Thermo Fisher Scientific) was used to detect apoptotic cells. Transfected PC9 or PC9R cells were seeded in 6-well plates $\left(5 \times 10^{5}\right.$ cells/well $)$ and treated with $1 \mu \mathrm{M}$ gefitinib. The cells were digested with trypsin-EDTA (Thermo Fisher Scientific), washed three times with PBS, resuspended in $500 \mu \mathrm{l}$ binding buffer, and then incubated with $5 \mu \mathrm{l}$ FITCconjugated annexin-V and $3 \mu \mathrm{PI}$ for $15 \mathrm{~min}$ at room temperature in the dark. Stained cells were sorted on a FACS Aria II flow cytometer (BD Biosciences, Franklin Lakes, NJ, USA).

\section{Cell cycle analysis}

Transfected PC9R cells were seeded in 6-well plates $\left(5 \times 10^{5}\right.$ cells/well $)$ and treated with $1 \mu \mathrm{M}$ gefitinib. The cells were collected, washed with PBS, and fixed in $70 \%$ ethanol for $24 \mathrm{~h}$ at $4^{\circ} \mathrm{C}$, then stained with PI in the dark for $30 \mathrm{~min}$ at room temperature. Cell cycle distribution was analysed by flow cytometry.

\section{Measurement of mitochondrial membrane potential}

The MitoProbe JC-1 assay kit (Thermo Fisher Scientific) was used according to the manufacturer's instructions to detect changes in mitochondrial membrane potential. JC-1 forms J-aggregates emitting red fluorescence at $590 \mathrm{~nm}$ in intact mitochondria, and J-monomers emitting green fluorescence at $490 \mathrm{~nm}$ in depolarized mitochondria; an increase in the J-monomer fraction indicates mitochondrial damage. The results of the assay were obtained by flow cytometry. 


\section{Measurement ROS level}

ROS were detected with the CellROX Orange probe (Invitrogen), which exhibits orange fluorescence upon oxidation by ROS. Transfected PC9R cells were seeded in 6-well plates $\left(5 \times 10^{5} /\right.$ well $)$ and treated with $1 \mu \mathrm{M}$ gefitinib. Cells were digested with trypsin-EDTA, incubated with $5 \mu \mathrm{M}$ CellROX Orange reagent at $37^{\circ} \mathrm{C}$ for $30 \mathrm{~min}$, washed twice with PBS, and analysed by flow cytometry to quantify ROS.

\section{qRT-PCR}

Total RNA was extracted using TRIzol reagent (Thermo Fisher Scientific) and reverse transcribed (Toyobo, Osaka, Japan) into cDNA, of which 20 ng were used as the template for qRT-PCR with the following forward and reverse primers: PLIN2, 5'-ACCAAGGTGCAGAGATGGAC-3' and 5'-CCAATTT AGGGTTGCCTAGC-3'; Survivin, 5'-AGAGTCCCTGGC TCCTCTA-3' and 5'-CCCGTTTCCCCAATGACTTA-3'; TOP2A, 5'-AGCAGATTAGCTTCGTCAACAGC-3' and 5'-ACATGTCTGCCGCCCTTAGA-3'; CDK1, 5'-TAGC GCGGATCTACCATACC-3' and 5'-CATGGCTACCACT TGACCTG-3'; BCL2L1， 5'-AAACTGGGTCGCATT GTGG-3' and 5'-TCTCGGCTGCTGCATTGTTC-3'; and $\beta$-actin, 5'-CTGGCACCCAGCACAATG-3' and 5'-CCGAT CCACACGGAGTACTTG-3'. The cycling conditions (40 cycles) were $95^{\circ} \mathrm{C}$ for $15 \mathrm{~s}, 60^{\circ} \mathrm{C}$ for $15 \mathrm{~s}$, and $2^{\circ} \mathrm{C}$ for $45 \mathrm{~s}$. Gene expression levels were normalized to that of $\beta$-actin according to the cycle threshold $\left(2^{-\Delta \Delta C T}\right)$ method.

\section{Western blotting}

Total protein was extracted with radioimmunoprecipitation buffer (Beyotime Institute of Biotechnology, Shanghai, China) and separated on polyacrylamide gels, then transferred to a polyvinylidene difluoride membrane that was incubated overnight at $4^{\circ} \mathrm{C}$ with antibodies again PLIN2, EGFR, p-Akt (Ser473), survivin, cleaved caspase-3 (Asp175), cleaved PARP (Asp214), and actin (all from Cell Signaling Technology, Danvers, MA, USA) followed by incubation with horseradish peroxidase-conjugated goat anti-rabbit or anti-mouse immunoglobulin $\mathrm{G}$ at room temperature for $1 \mathrm{~h}$. The proteins were visualized by enhanced chemiluminescence (Pierce, Rockford, IL, USA) and band intensity was analysed with Quantity One v.4.6 software (Bio-Rad, Hercules, CA, USA).

\section{cDNA array and signalling pathway analysis}

Total RNA was extracted from PC9R cells transfected with shPLIN2-1 or negative control shRNA using the RNeasy Plus Mini kit (Qiagen, Valencia, CA, USA) and reverse transcribed into cDNA, which was amplified using a OneArray plus RNA amplification kit (Phalanx Biotech Group, Taiwan). Cy5-labelled RNA targets were hybridized to the Human Whole Genome OneArray (Phalanx Biotech Group). Signal intensity was measured with an Agilent Microarray Scanner (G2505C; Agilent Technologies, Santa Clara, CA, USA) and analysed with the Resolver system (Rosetta Biosoftware, Seattle, WA, USA). Statistical analyses were performed using the modeling techniques provided by the software.

\section{Tumourigenicity assay}

Animal experiments were approved by the Institutional Animal Care and Use Committee at Zhongshan Hospital of Fudan University, China. Male BALB/c nude mice (4-6 weeks old) were obtained from the Shanghai Experimental Animal Center of the Chinese Academy of Sciences (Shanghai, China) and were subcutaneously injected into the right flank with PC9R cells. Tumour volume was calculated according to the following formula: volume $=\left(\right.$ length $\times$ width $\left.^{2}\right) / 2$. After 1 month, tumours were dissected and weighed for quantification. Ki67 immunohistochemistry was performed to detect proliferating cells in paraffin sections of xenograft tumours. Ki67-positive cells in randomly selected fields from each tissue section were quantified using ImageJ software.

\section{Pleural effusion sample collection}

A total of 26 lung adenocarcinoma patients, including 17 who were sensitive to and nine who had acquired resistance to EGFR TKIs, were enrolled in the study at the Department of Pulmonary Medicine, Zhongshan Hospital, Fudan University. All study participants provided informed consent and the study protocol was approved by the institutional ethics committee (approval number B2016154R). All patient managements and pleural effusion sample collection were carried out in accordance with the relevant guidelines. Pleural effusions were collected and centrifuged at $1000 \times g$ for $10 \mathrm{~min}$. Cell pellets were washed twice with PBS and total RNA was extracted from cells using TRIzol reagent (Invitrogen). PLIN2 mRNA level was quantitated by qRT-PCR.

\section{Statistical analysis}

Experiments were performed with triplicate samples. Data are expressed as mean $\pm \mathrm{SD}$ of at least three independent experiments. Differences among groups were evaluated by one-way analysis of variance followed by Bonferroni's multiple comparisons test, while the Student's $t$ test was used to compare two different groups. $P<0.05$ was considered statistically significant.

\section{Author contributions}

Jian Zhou, Chunxue Bai and Yuanlin Song supervised the conduction of the entire project. Meijia 
Chang, Jie Hu, Tao Fang, and Jing Li performed the research, analyzed the data, and prepared the manuscript. All authors read and approved the final manuscript.

\section{CONFLICTS OF INTEREST}

All authors have no financial, professional or personal conflicts to disclose.

\section{FUNDING}

This work was supported by the State Key Basic Research Program (973) project (2015CB553404), Shanghai Municipal Leading Academic Discipline Project No. B115, Zhongshan Hospital Clinical Research Foundation No.2016ZSLC05, National Basic Research Program of China (973 Program) No. 2012CB933304, National key R\&D plan No. 2016YFC1304104, Natural Science Foundation of China No.81400018, No.81570028, No.81770039, Natural Science Foundation of Shanghai No.15ZR1406400, and the Shanghai Three-Year Plan of the Key Subjects Construction in Public HealthInfectious Diseases and Pathogenic Microorganism No. 15GWZK0102.

\section{REFERENCES}

1. Metro G, Crinò L. Advances on EGFR mutation for lung cancer. Transl Lung Cancer Res. 2012; 1:5-13.

2. Lee DH. Treatments for EGFR-mutant non-small cell lung cancer (NSCLC): The road to a success, paved with failures. Pharmacol Ther. 2017; 174:1-21.

3. Maemondo M, Inoue A, Kobayashi K, Sugawara S, Oizumi $\mathrm{S}$, Isobe $\mathrm{H}, \mathrm{Gemma} \mathrm{A}$, Harada M, Yoshizawa H, Kinoshita I, Fujita Y, Okinaga S, Hirano H, et al, and North-East Japan Study Group. Gefitinib or chemotherapy for non-small-cell lung cancer with mutated EGFR. N Engl J Med. 2010; 362:2380-2388.

4. Rosell R, Carcereny E, Gervais R, Vergnenegre A, Massuti B, Felip E, Palmer o R, Garcia-Gomez R, Pallares C, Sanchez JM, Porta R, Cobo M, Garrido P, et al, and Spanish Lung Cancer Group in collaboration with Groupe Français de Pneumo-Cancérologie and Associazione Italiana Oncologia Toracica. Erlotinib versus standard chemotherapy as first-line treatment for European patients with advanced EGFR mutation-positive non-small-cell lung cancer (EURTAC): a multicentre, open-label, randomised phase 3 trial. Lancet Oncol. 2012; 13:239-246.

5. Camidge DR, Pao W, Sequist LV. Acquired resistance to TKIs in solid tumours: learning from lung cancer. Nat Rev Clin Oncol. 2014; 11:473-481.

6. Engelman JA, Zejnullahu K, Mitsudomi T, Song Y, Hyland C, Park JO, Lindeman N, Gale CM, Zhao X, Christensen J, Kosaka T, Holmes AJ, Rogers AM, et al. MET amplification leads to gefitinib resistance in lung cancer by activating ERBB3 signaling. Science. 2007; 316:1039-1043.

7. Yano S, Yamada T, Takeuchi S, Tachibana K, Minami Y, Yatabe Y, Mitsudomi T, Tanaka H, Kimura T, Kudoh S, Nokihara H, Ohe Y, Yokota J, et al. Hepatocyte growth factor expression in EGFR mutant lung cancer with intrinsic and acquired resistance to tyrosine kinase inhibitors in a Japanese cohort. J Thorac Oncol. 2011; 6:2011-17.

8. Conte M, Franceschi C, Sandri M, Salvioli S. Perilipin 2 and age-related metabolic diseases: a new perspective. Trends Endocrinol Metab. 2016; 27:893-903.

9. Fujimoto M, Matsuzaki I, Yamamoto Y, Yoshizawa A, Warigaya K, Iwahashi Y, Kojima F, Furukawa F, Murata SI. Adipophilin expression in cutaneous malignant melanoma. J Cutan Pathol. 2017; 44:228-236.

10. Matsubara J, Honda K, Ono M, Sekine S, Tanaka Y, Kobayashi M, Jung G, Sakuma T, Nakamori S, Sata $\mathrm{N}$, Nagai H, Ioka T, Okusaka T, et al. Identification of adipophilin as a potential plasma biomarker for colorectal cancer using label-free quantitative mass spectrometry and protein microarray. Cancer Epidemiol Biomarkers Prev. 2011; 20:2195-2203.

11. Qiu B, Ackerman D, Sanchez DJ, Li B, Ochocki JD, Grazioli A, Bobrovnikova-Marjon E, Diehl JA, Keith B, Simon MC. HIF2 alpha-dependent lipid storage promotes endoplasmic reticulum homeostasis in clear-cell renal cell carcinoma. Cancer Discov. 2015; 5:652-667.

12. Fujimoto M, Yoshizawa A, Sumiyoshi S, Sonobe M, Menju T, Hirata M, Momose M, Date H, Haga H. Adipophilin expression in lung adenocarcinoma is associated with apocrine-like features and poor clinical prognosis: an immunohistochemical study of 328 cases. Histopathology. 2017; 70:232-241.

13. Chang TH, Tsai MF, Su KY, Wu SG, Huang CP, Yu SL, Yu YL, Lan CC, Yang CH, Lin SB, Wu CP, Shih JY, Yang PC. Slug confers resistance to the epidermal growth factor receptor tyrosine kinase inhibitor. Am J Respir Crit Care Med. 2011; 183:1071-1079.

14. Pao W, Miller V, Zakowski M, Doherty J, Politi K, Sarkaria I, Singh B, Heelan R, Rusch V, Fulton L, Mardis E, Kupfer D, Wilson R, et al. EGF receptor gene mutations are common in lung cancers from "never smokers" and are associated with sensitivity of tumors to gefitinib and erlotinib. Proc Natl Acad Sci U S A. 2004; 101:13306-13311.

15. Tan CS, Gilligan D, Pacey S. Treatment approaches for EGFR-inhibitor-resistant patients with non-small-cell lung cancer. Lancet Oncol. 2015; 16:e447-e459.

16. Listenberger LL, Ostermeyer-Fay AG, Goldberg EB, Brown WJ, Brown DA. Adipocyte differentiation-related protein reduces the lipid droplet association of adipose triglyceride lipase and slows triacylglycerol turnover. J Lipid Res. 2007; 48:2751-2761. 
17. Robey RB, Weisz J, Kuemmerle NB, Salzberg AC, Berg A, Brown DG, Kubik L, Palorini R, Al-Mulla F, Al-Temaimi R, Colacci A, Mondello C, Raju J, et al. Metabolic reprogramming and dysregulated metabolism: cause, consequence and/or enabler of environmental carcinogenesis? Carcinogenesis. 2015; 36:S203-S231.

18. Merino Salvador M, Gómez de Cedrón M, Moreno Rubio J, Falagán Martínez S, Sánchez Martínez R, Casado E, Ramírez de Molina A, Sereno M. Lipid metabolism and lung cancer. Crit Rev Oncol Hematol. 2017; 112:31-40.

19. Jia Y, Yun CH, Park E, Ercan D, Manuia M, Juarez J, Xu C, Rhee K, Chen T, Zhang H, Palakurthi S, Jang J, Lelais G, et al. Overcoming EGFR(T790M) and EGFR(C797S) resistance with mutant-selective allosteric inhibitors. Nature. 2016; 534:129-132.

20. Costa DB, Halmos B, Kumar A, Schumer ST, Huberman MS, Boggon TJ, Tenen DG, Kobayashi S. BIM mediates EGFR tyrosine kinase inhibitor-induced apoptosis in lung cancers with oncogenic EGFR mutations. PLoS Med. 2007; 4:1669-1679.

21. Kuwano M, Sonoda K, Murakami Y, Watari K, Ono M. Overcoming drug resistance to receptor tyrosine kinase inhibitors: learning from lung cancer. Pharmacol Ther. 2016; 161:97-110.

22. Ohashi K, Sequist LV, Arcila ME, Moran T, Chmielecki J, Lin YL, Pan Y, Wang L, de Stanchina E, Shien K, Aoe K, Toyooka S, Kiura K, et al. Lung cancers with acquired resistance to EGFR inhibitors occasionally harbor BRAF gene mutations but lack mutations in KRAS, NRAS, or MEK1. Proc Natl Acad Sci U S A. 2012; 109:E2127-E2133.

23. Fregoso Lomas M, De Vito S, Boisclair Lachance JF, Houde J, Nilson LA. Determination of EGFR signaling output by opposing gradients of BMP and JAK/STAT activity. Curr Biol. 2016; 26:2572-2582.

24. Pérez-Ramírez C, Cañadas-Garre M, Molina MA, FausDáder MJ, Calleja-Hernández MA. PTEN and PI3K/AKT in non-small-cell lung cancer. Pharmacogenomics. 2015; 16:1843-1862.

25. Okamoto K, Okamoto I, Okamoto W, Tanaka K, Takezawa K, Kuwata K, Yamaguchi H, Nishio K, Nakagawa K. Role of survivin in EGFR inhibitor-induced apoptosis in non-small cell lung cancers positive for EGFR mutations. Cancer Res. 2010; 70:10402-10410. 